\title{
Public Awareness and Perceptions about Diabetes in the State of Qatar
}

Al-Anoud Al-Thani ${ }^{1}$, Aiman H. Farghaly ${ }^{1}$, Hammad Akram ${ }^{1}$, Shams Eldin Khalifa ${ }^{1}$, Benjamin Vinodson ${ }^{1}$ , Alma M. Loares ${ }^{1}$, Abdul-Badi Abou-Samra ${ }^{2}$

1. Ministry of Public Health, State of Qatar, Doha, QAT 2. Department of Medicine, Hamad Medical Corporation

Corresponding author: Aiman H. Farghaly, aimanfarghaly@yahoo.com

\section{Abstract}

\section{Introduction}

Diabetes is a well-known global public health challenge affecting millions globally. The aims of this study are to examine the community diabetes knowledge, perceptions, and awareness among the public in Qatar regarding (1) disease symptoms, risk factors, complications, prevention, and associated behaviors, and (2) local diabetes campaigns and available services or resources.

\section{Methods}

This study involved a total of 501 respondents selected through purposive sampling from major public malls and public places in Doha, Qatar between February and May 2015. Data were gathered by face-to-face interview utilizing a semi-structured questionnaire. Results were analyzed using count, percentage, median, chi-square test, z-test on proportion and logistic regression. The analysis was carried out at $5 \%$ level of significance using SPSS version 22 (IBM Corporation, Chicago, IL, USA).

\section{Results}

About $92 \%$ of participants knew at least one type of diabetes. Over $43.9 \%$ were physically active for $1-3$ days per week. Highest proportion of the population perceived blindness (86\%) as the top complication; frequent urination (58\%) and excessive thirst (41\%) as primary symptoms; hereditary factors (74\%) and obesity (74\%) as the top risk factors; and exercise (77\%) and diet (72\%) as among the preventive measures. Demographic overview of diabetes and lifestyle factors showed that the odds of obtaining screening tests were higher among females ( $\mathrm{OR}=1.7, \mathrm{P}=0.003)$, age $35-45(\mathrm{OR}=2.2, \mathrm{P}=0.003)$, age $\geqslant 55(\mathrm{OR}=4.1, \mathrm{P}<0.001)$ and married $(\mathrm{OR}=3.0, \mathrm{CI}=2.0-4.6, \mathrm{P}<0.001)$ compared with their respective counterpart groups.

\section{Conclusions}

The general population in Qatar require more awareness and education about diabetes prevention and associated risk factors. This could be achieved by implementation of more public health campaigns to

Received 04/26/2018

Review began 04/27/2018 Review ended 05/08/2018 Published 05/22/2018

() Copyright 2018

Al-Thani et al. This is an open access article distributed under the terms of the Creative Commons Attribution License CC-BY 3.0., which permits unrestricted use, distribution, and reproduction in any medium, provided the original author and source are credited.
Categories: Preventive Medicine, Public Health, Epidemiology/Public Health Keywords: diabetes, diabetes awareness, qatar, public perception

\section{Introduction}

Diabetes is a well-known global public health challenge affecting over 400 million people worldwide. Despite the awareness of the problem and technological advancements in healthcare systems, the adult diabetes prevalence has almost doubled since 1980 worldwide. Diabetes is a huge financial burden because of the associated multi-organ complications among sufferers and a major cause of mortality around the world [1]. The Arab world is not spared by the diabetes epidemic as well, for example in the Eastern Mediterranean region countries the prevalence of this disease ranges from $3.5 \%$ to as high as $30 \%$ [2].

The State of Qatar is a country located in the Gulf Cooperation Council region adjacent to Arabian Gulf with over 2.5 million population. The country has encountered a rapid socio-economic development in recent past few decades that led to a change in lifestyles of the population influenced by things such as easily available fast food, increased use of personal vehicles for transportation, increased screen time and fewer chances of being physically active etc. Diabetes and associated risk factors such as obesity and lack of physical activity are now considered major public health challenges in Qatar [3-4].

Qatar's National Health Strategy (NHS) envisions to encourage a healthy lifestyle, promote public health and ensure that quality healthcare services are accessible to the community [5]. With the implementation of one of its strategies, the National Diabetes Strategy, which aims to increase public awareness about diabetes, promote prevention and support healthy lifestyles, NHS aspires to decrease the incidence and 
complications of diabetes for better health and quality of life in Qatar [6]. This is important since according to a national survey in Qatar that was conducted in 2012, the diabetes prevalence among Qatari nationals was $16.7 \%$ which is higher than the prevalence identified in a 2006 survey. In the same survey, 11.6\% Qatari nationals, 6.6\% non-Qatari residents composed of Arab, Asian and Western nationals, and $8.3 \%$ of the total sample had self-reported diabetes or were treated with medicines for it [7-8]. The survey results have also indicated that certain behavioral and social factors have an impact on obesity as well as on diabetes prevalence among population $[7,9]$.

In 2015, the Ministry of Public Health (MoPH), Qatar with the assistance of YouGov (a market research organization) performed a public survey named “Diabetes Awareness and Perception in Qatar” to assess the knowledge of community pertaining to diabetes and associated factors. The survey was an important initiative to further strengthen the existing national strategy that addresses various non-communicable diseases (NCDs) and promote prevention, monitoring and community education related to diabetes as well.

The present study provides an overview of the results of the above-mentioned survey and examines the community perception and awareness about diabetes and related factors such as diabetes symptoms, risk factors, complications, prevention, and lifestyle behaviors. The study further explores the relationship of demographic factors with diabetes screening and selected behavioral factors.

\section{Materials And Methods}

\section{Sampling}

The study was carried out at public places with the general population, such as malls and shopping centers. A total of 501 individuals were interviewed face-to-face for about 20-30 minutes between February and May 2015 . This sample provided a margin of error of $\pm 4.4 \%$ at $95 \%$ confidence interval. The target population was 16 years or older and included both genders from different nationalities categorized as Qatari, Arab expats, Asian expats, and Western expats. The sample selection was achieved by using purposive sampling method, with inclusion criteria based on quotas per gender, age groups, and nationality (Table 1). Interviews were conducted as per accordance with the sampling plan in which an equal number of Qatari and non-Qatari citizens and an equal number of male and female participants were selected. Age groups were categorized into $16-24$ years, $25-34$ years, $35-54$ years and 55 years or older (>54). The sampling proportion of each age group was selected to correspond approximately to the estimated 2015 Qatar census proportion within the same age groups. A sample of 501 was achieved based on the availability of budget, resources and associated logistics.

\begin{tabular}{|c|c|c|c|c|c|}
\hline \multirow[b]{2}{*}{ Age } & \multicolumn{2}{|l|}{ Qataris } & \multicolumn{2}{|l|}{ Expats } & \multirow[t]{2}{*}{ Iotal } \\
\hline & Males & Females & Males & Females & \\
\hline $16-24$ & 25 & 25 & 20 & 15 & 85 \\
\hline $25-34$ & 40 & 40 & 60 & 35 & 175 \\
\hline $35-54$ & 40 & 40 & 60 & 36 & $1 / 5$ \\
\hline$>54$ & 20 & 20 & 15 & 10 & 65 \\
\hline TOTAL & 250 & & 250 & & 500 \\
\hline
\end{tabular}

TABLE 1: Proposed Sample Quota for Survey, By Gender, Nationality and Age Groups

In addition to the demographic and socio-economic information of participants, the survey questions were designed to obtain their perception, awareness and knowledge related to diabetes. The participants were able to share their awareness about local campaigns and the mode of related messages transmitted to them, knowledge about the types, risk factors, symptoms, complications of diabetes and understanding of preventive factors. Furthermore, the survey also covered participant's personal behaviors in terms of physical activity and diet.

\section{Analysis}

Statistical analyses were performed using the SPSS software version 22.0 (IBM Corporation, Chicago, IL, USA). The results were reported as sample size and/or percentage with $95 \%$ confidence interval while awareness about complications of diabetes was measured using a 5-point Likert scale responses [1-strongly disagree, 2- disagree, 3-neither disagree nor agree (neutral), 4-agree, 5-strongly agree] and generally assessed using median value. Chi-square test was performed to determine the association between gender, and variables assessing diabetes awareness and healthy lifestyle. Again, Z test on proportion was used to 


\section{Cureus}

evaluate the differences in proportions by gender. The effect of various demographic factors on diabetes screening, frequencies of exercises, and healthy eating habits was assessed by logistic regression, which computed odds ratio and 95\% confidence interval. Level of significance was given at $\mathrm{P}<0.05$.

\section{Ethical procedures}

Ethical procedures were followed during the survey implementation and data handling procedures. The consent was obtained from the participants before the survey was carried out. The collected information was only used for the scientific purposes without breaching any personal or confidential information. Parental consent was sought for the respondents aged below 18 years.

\section{Results}

\section{Characteristics of Participants}

A total of 501 participants of ages 16 years or older were interviewed. Based on the selection criteria, about equal numbers of Qatari and non-Qatari nationals were included (48\% and 52\%, respectively); and about equal numbers of male and female subjects ( $51.7 \%$ and $48.3 \%$, respectively). Highest number of participants were in the age group $24-34$ years (170, 33.9\%) followed by $35-54$ years (161, 32.1\%), 16-24 years (110, 22\%) and $55+(60,12 \%)$ respectively. About one-half of all participants $(50.9 \%)$ reported being married with children. The characteristics of participants are shown in Table 2. 


\section{Cureus}

\section{General characteristics}

N

Age

16-24 years

$110(22.0)$

25-34 years

170 (33.9)

35-54 years

$161(32.1)$

$55+$ years

$60(12.0)$

Gender

Male

Female

Nationality group

Qatari

Arab

Asian

Westerner

57 (11.4)

Married status

Single

179 (35.7)

Married with children

255 (50.9)

Married without children

51 (10.2)

Widowed/ divorced

16 (3.2)

Monthly Income

1,500-9,999 QAR

96 (19.2)

10,000-19,999 QAR

$128(25.5)$

20,000+ QAR

$214(42.7)$

Don't know/ can't say

$63(12.6)$

\section{TABLE 2: Characteristics of Study Population}

\section{Diabetes Awareness and Screening}

The respondents were asked if they were aware of different types of diabetes. The first type of diabetes reported by each respondent was recorded under one variable. Then the second response was recorded as a separate variable using the question "What other types of diabetes are you aware of" (data not shown in this article). As a first response, $92 \%$ were able to provide a type of diabetes while 38 (7.6\%) stated that they did not know any type(s). A total of 61 (12.2\%) respondents stated that they were diagnosed with diabetes $(10 \%$ men, $14.5 \%$ women, $17 \%$ Qatari, $8 \%$ non-Qatari), $6.2 \%$ also had someone in their family or friends with diabetes, and about $45 \%$ stated that someone close to them had been diagnosed with diabetes (Table 3).

\begin{tabular}{|c|c|c|c|}
\hline $\mathrm{N}$ & 501 & 259 & 242 \\
\hline \multicolumn{4}{|l|}{ Diagnosed with diabetes } \\
\hline Diagnosed with diabetes (myself) & $61(12.2)$ & $26(10.0)$ & 35 (14.5) \\
\hline
\end{tabular}




\section{Cureus}

Diagnosed with diabetes myself and members of family/ friends

Someone close to me who has been diagnosed with diabetes

Neither I nor family / friends have been diagnosed with diabetes

Awareness based on type of diabetes

Pre-diabetes

Type 1 Diabetes

Type 2 Diabetes

Gestational diabetes

None of the above

Ever Screened for Diabetes

Yes

No

Ways to prevent Diabetes ${ }^{\text {a }}$

Frequent exercise

Healthy Diet

Maintain a healthy weight

Get checked

Quit smoking

Top 5 perceived signs of diabetes ${ }^{a}$

Frequent urination, particularly at night

Excessive thirst

Tiredness

Distorted vision

Tingling/ Numbness of hands and feet

Preferred location for diabetes check in case of signs of diabetes ${ }^{\mathrm{a}}$

Public Hospital

Private hospital

Private clinic

Diabetes care center

As home with a home test kit

Perceived top risk factors of diabetes ${ }^{a}$

Hereditary

Injury or disease of the pancreas \& insulin

Obesity

Lack of exercise

Poor Diet

High blood glucose

Top 5 sources of ad or campaign about diabetes ${ }^{a}$

TV ad

$\begin{array}{lll}31(6.2) & 18(6.9) & 13(5.4) \\ 225(44.9) & 107(41.3) & 118(48.8) \\ 184(36.7) & 108(41.7) & 76(31.4)\end{array}$

$131(26.1) \quad 78(30.1) \quad 53(21.9) \ddagger$

$204(40.7) \quad 117(45.2) \quad 87(36.0)$

$69(13.8) \quad 43(16.6) \quad 26(10.7)$

$59(11.8) \quad 5(1.9) \quad 54(22.3)$

$38(7.6) \quad 16(6.2) \quad 22(9.1)$

$184(36.7) \quad 79(30.5) \quad 105(43.4) \ddagger$

$317(63.3) \quad 180(69.5) \quad 137(56.6)$

$386(77.0) \quad 208(80.3) \quad 178(73.6)$

$363(72.5) \quad 199(76.8) \quad 164(67.8)^{\star}$

$301(60.1) \quad 150(57.9) \quad 151(62.4)$

$302(60.3) \quad 152(58.7) \quad 150(62.0)$

$100(20.0) \quad 60(23.2) \quad 40(16.5)$

$291(58.1) \quad 158(61.0) \quad 133(55.0)$

$205(40.9) \quad 112(43.2) \quad 93(38.4)$

$174(34.7) \quad 87(33.6) \quad 87(36.0)$

$162(32.3) \quad 84(32.4) \quad 78(32.2)$

$152(30.3) \quad 77(29.7) \quad 75(31.0)$

$281(56.1) \quad 145(56.0) \quad 136(56.2)$

$178(35.5) \quad 103(39.8) \quad 75(31.0)^{*}$

$147(29.3) \quad 92(35.5) \quad 55(22.7)^{*}$

$203(40.5) \quad 110(42.5) \quad 93(38.4)$

$141(28.1) \quad 62(23.9) \quad 79(32.6)^{*}$

$\begin{array}{lll}370(73.9) & 200(77.2) & 170(70.2) \\ 314(62.7) & 162(62.5) & 152(62.8) \\ 370(73.9) & 198(76.4) & 172(71.1) \\ 309(61.7) & 164(63.3) & 145(59.9) \\ 307(61.3) & 163(62.9) & 144(59.5) \\ 305(60.9) & 159(61.4) & 146(60.3) \\ 54(35.3) & 28(35.9) & 26(34.7)\end{array}$




\section{Cureus}

\begin{tabular}{|c|c|c|c|}
\hline Social media & $32(20.9)$ & $18(23.1)$ & $14(18.7)$ \\
\hline Internet & $30(19.6)$ & $16(20.5)$ & $14(18.7)$ \\
\hline Billboard & $60(39.2)$ & $33(42.3)$ & $27(36.0)$ \\
\hline At public hospital/ clinic & $38(24.8)$ & $20(25.6)$ & $18(24.0)$ \\
\hline \multicolumn{4}{|l|}{ Organizations communicated diabetes awareness } \\
\hline Action of diabetes & $18(11.8)$ & $10(12.8)$ & $8(10.7)$ \\
\hline Qatar Diabetes association & $34(22.2)$ & $16(20.5)$ & $18(24.0)$ \\
\hline Ministry of Public Health & $44(28.8)$ & $23(29.5)$ & $21(28.0)$ \\
\hline Major Community Hospital (Hamad Medical Corporation) & $51(33.3)$ & $29(37.2)$ & $22(29.3)$ \\
\hline
\end{tabular}

\section{TABLE 3: Participant's Awareness Pertaining to Diabetes}

Overall, 36.7\% stated that they were screened for diabetes with a higher percentage among women (43.4\%) compared with men (30.5\%). Most common location to obtain diabetes screening was diabetes clinic (34.2\%) followed by the primary health care centers (29.3\%). The top reason to obtain screening was physician or nurse referral (28\%) followed by the convenience of getting screening (26\%) (Figure 1). Among Qatari nationals, the top reason for obtaining screening (34\%) was the referral from a doctor or a nurse. For nonQataris, the top reason for getting screening was family or friends recommendations (23.8\%). Those who were not screened, 59.6 Qatari and $48.9 \%$ non-Qatari, stated that the main reason for not obtaining screening was that they never thought about it (Figure 2). This was also the main reason for the overall sample (Figure 1). When participants were asked if they know the ways to prevent diabetes, the majority considered the frequency of exercise (77\%) followed by healthy diet (72.5\%) and maintaining a healthy weight (60\%) as preventive measures (Table 3). Regarding the signs and symptoms of diabetes, over $58 \%$ of participants mentioned the frequency of urination especially at night is the main sign followed by excessive thirst (41\%) and tiredness (34.7\%). The respondents considered obesity (73.9\%) and hereditary factors (73.9\%) as the top risk factors in the development of diabetes followed by almost equal responses (60\%) for pancreatic disorders, poor diet, lack of exercise, getting older, and high blood glucose. Billboards (39.2\%) were considered by respondents as the main source of diabetes campaigns advertisement followed by television (35.3\%) (Table 3).

\section{Top 5 factors influencing the diabetes screening by gender}

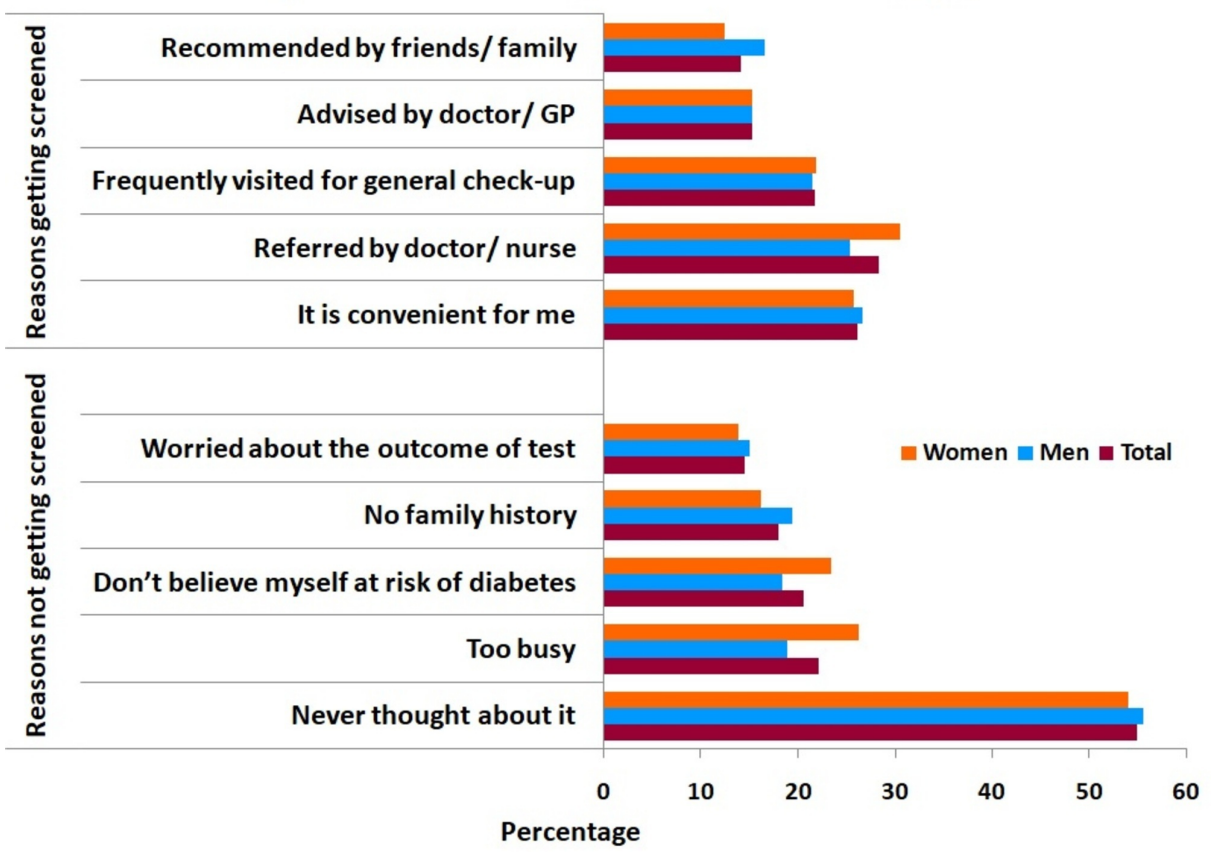

FIGURE 1: Top 5 Influencing Factors for Diabetes Screening Among 


\section{Cureus}

Participants by Gender

Top 5 factors influencing the diabetes screening by nationality

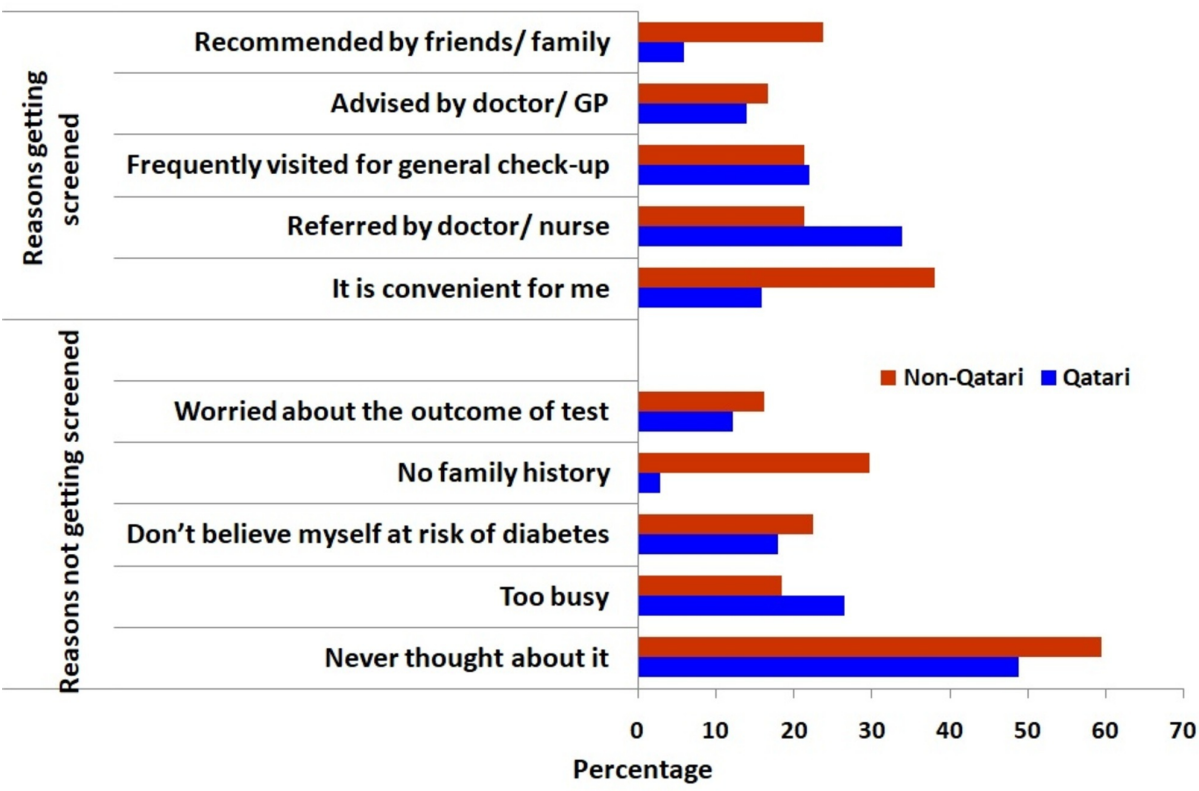

FIGURE 2: Top 5 Influencing Factors for Diabetes Screening Among Participants by Nationality

Perceptions about diabetes and respondents' awareness on diabetes complications were likewise determined as shown in Table 4. Except for one, all of the questions evaluating the diabetes awareness derived a median value of 4 , which indicates that half of the respondents agreed to the statements mentioned. Salient to the findings was that majority of the participants had highest awareness on blindness as a complication of diabetes, as more than half strongly agreed that diabetes causes blindness, having a median value of 5 . 


\begin{tabular}{|c|c|c|c|c|c|c|}
\hline & $\begin{array}{l}\text { Strongly } \\
\text { disagree \% } \\
\text { (Cl\%) }\end{array}$ & $\begin{array}{l}\text { Disagree } \\
\% \text { (CI\%) }\end{array}$ & $\begin{array}{l}\text { Neutral } \\
\%(\mathrm{Cl} \%)\end{array}$ & $\begin{array}{l}\text { Agree } \\
\% \text { (Cl\%) }\end{array}$ & $\begin{array}{l}\text { Strongly } \\
\text { agree \% (Cl\%) }\end{array}$ & Median \\
\hline $\begin{array}{l}\text { Advanced screening tools available to detect } \\
\text { diabetes }\end{array}$ & $0.4(0.1,1.4)$ & $\begin{array}{l}1.0(0.4, \\
2.3)\end{array}$ & $\begin{array}{l}23.2(19.7, \\
27.0)\end{array}$ & $\begin{array}{l}31.5 \\
(27.6, \\
35.7)\end{array}$ & $\begin{array}{l}43.9(39.6, \\
48.3)\end{array}$ & 4 \\
\hline $\begin{array}{l}\text { People with diabetes have a higher risk of } \\
\text { developing infections }\end{array}$ & $2.0(1.0,3.6)$ & $\begin{array}{l}7.4(5.4 \\
10.0)\end{array}$ & $\begin{array}{l}26.1(22.5, \\
30.1)\end{array}$ & $\begin{array}{l}48.3 \\
(43.9, \\
52.7)\end{array}$ & $\begin{array}{l}16.2(13.2, \\
19.6)\end{array}$ & 4 \\
\hline Diabetes is a leading cause of CVD & $3.9(2.6,6.1)$ & $\begin{array}{l}8.6(6.4 \\
11.4)\end{array}$ & $\begin{array}{l}20.8(17.4, \\
24.5)\end{array}$ & $\begin{array}{l}31.7 \\
(27.8 \\
35.9)\end{array}$ & $\begin{array}{l}34.9(30.8, \\
39.2)\end{array}$ & 4 \\
\hline Diabetes can cause blindness & $0.2(0.04,1.1)$ & $\begin{array}{l}1.0(0.4, \\
2.3)\end{array}$ & $\begin{array}{l}12.4(9.8, \\
15.6)\end{array}$ & $\begin{array}{l}28.3 \\
(24.6, \\
32.4)\end{array}$ & $\begin{array}{l}58.1(53.7 \\
62.3)\end{array}$ & 5 \\
\hline $\begin{array}{l}\text { Kidney failure is a serious complication of } \\
\text { diabetes }\end{array}$ & $0.4(0.1,1.4)$ & $\begin{array}{l}4.6(3.1, \\
6.8)\end{array}$ & $\begin{array}{l}25.9(22.3, \\
29.9)\end{array}$ & $\begin{array}{l}28.9 \\
(25.1, \\
33.1)\end{array}$ & $\begin{array}{l}40.1(35.9, \\
44.8)\end{array}$ & 4 \\
\hline $\begin{array}{l}\text { People with diabetes carry a high risk of } \\
\text { amputation }\end{array}$ & $0.4(0.1,1.4)$ & $\begin{array}{l}8.8(6.6, \\
11.6)\end{array}$ & $\begin{array}{l}19.8(16.5, \\
23.5)\end{array}$ & $\begin{array}{l}38.5 \\
(34.4, \\
42.8)\end{array}$ & $\begin{array}{l}32.5(28.6, \\
36.7)\end{array}$ & 4 \\
\hline $\begin{array}{l}\text { Diabetes can cause pregnancy complications in } \\
\text { women with any type of diabetes }\end{array}$ & $0.2(0.04,1.1)$ & $\begin{array}{l}3.4(2.1 \\
5.4)\end{array}$ & $\begin{array}{l}23.8(20.2, \\
27.7)\end{array}$ & $\begin{array}{l}37.7 \\
(33.6, \\
42.0)\end{array}$ & $\begin{array}{l}34.9(30.9, \\
39.2)\end{array}$ & 4 \\
\hline Type 1 diabetes cannot be prevented & $0.4(0.1,1.4)$ & $\begin{array}{l}7.2(5.2, \\
9.8)\end{array}$ & $\begin{array}{l}28.1(24.4, \\
32.2)\end{array}$ & $\begin{array}{l}30.5 \\
(26.7, \\
34.7)\end{array}$ & $\begin{array}{l}33.7(29.7 \\
37.9)\end{array}$ & 4 \\
\hline $\begin{array}{l}\text { Healthy life-style choices can help prevent type } \\
2 \text { diabetes }\end{array}$ & $0.6(0.2,1.8)$ & $\begin{array}{l}4.2(2.7, \\
6.3)\end{array}$ & $\begin{array}{l}20.8(17.4, \\
24.5)\end{array}$ & $\begin{array}{l}36.1 \\
(32.0, \\
40.4)\end{array}$ & $\begin{array}{l}38.3(34.2, \\
42.6)\end{array}$ & 4 \\
\hline $\begin{array}{l}\text { Blood sugar should be checked at least once a } \\
\text { year }\end{array}$ & $8.4(6.2,11.1)$ & $\begin{array}{l}7.0(5.1, \\
9.6)\end{array}$ & $\begin{array}{l}19.0(15.8, \\
22.6)\end{array}$ & $\begin{array}{l}39.9 \\
(35.7, \\
44.3)\end{array}$ & $\begin{array}{l}25.7 \text { (22.1, } \\
29.8)\end{array}$ & 4 \\
\hline
\end{tabular}

TABLE 4: The Respondent's Perceptions of Diabetes and Related Complications

\section{Lifestyle}

Over $43.9 \%$ of the respondents were physically active for $1-3$ days per week, $31.5 \%$ were engaged in it $2-3$ times per week and only $11 \%$ have a daily physical activity. Furthermore, $25 \%$ stated that they do not perform any exercise throughout the week. Most common place of the exercise was fitness centers (31\%) followed by public areas (21\%), homes (17\%) and sports clubs (15\%), respectively. About $57 \%$ of respondents considered their eating habits as healthy and $59.7 \%$ were satisfied or very satisfied with their eating habits. The family's persuasion had the highest impact on the change in eating habits of the respondents (59.7\%) followed by doctor's advice (48.9\%). The respondents stated that in order to keep a track of their health they measure their body weight (61.5\%), monitor calorie intake (43.5\%), assess fitness level (37.1\%) and/or use a health/fitness mobile application (29.1\%). On the contrary, about 7\% do not track their health (Table 5).

\begin{tabular}{|c|c|c|c|}
\hline \multirow[t]{2}{*}{ Healthy lifestyle attributes } & Total n (\%) & Men n (\%) & Women n (\%) \\
\hline & 501 & 259 & 242 \\
\hline \multicolumn{4}{|l|}{ Frequency of exercise (C1) } \\
\hline No exercise & $125(25.0)$ & $56(21.6)$ & $69(28.5)$ \\
\hline
\end{tabular}




\section{Cureus}

\begin{tabular}{|c|c|c|c|}
\hline 1-3 times a week & 220 (43.9) & $123(47.5)$ & $97(40.1)$ \\
\hline 4-7 times a week & $156(31.1)$ & $80(30.9)$ & $76(31.4)$ \\
\hline \multicolumn{4}{|l|}{ Place of exercise (C2) ${ }^{\mathrm{a}}$} \\
\hline Fitness center/ Gym & $118(31.0)$ & $75(36.9)$ & $43(24.8)$ \\
\hline Public areas & $79(21.0)$ & $41(20.2)$ & $38(22.0)$ \\
\hline Sports club & $55(15.0)$ & $28(13.8)$ & $27(15.6)$ \\
\hline At home & $64(17.0)$ & $27(13.3)$ & $37(21.4)$ \\
\hline Doha Corniche (city beachline) & $34(9.0)$ & $18(8.9)$ & $16(9.2)$ \\
\hline Public parks & $24(6.4)$ & $13(6.4)$ & $11(6.4)$ \\
\hline \multicolumn{4}{|c|}{ Consider Eating habits healthy? (C3) } \\
\hline Yes & $285(56.9)$ & $152(58.7)$ & $133(55.0)$ \\
\hline No & $181(36.1)$ & $89(34.4)$ & $92(38.0)$ \\
\hline Don't know & $35(7.0)$ & $18(6.9)$ & $17(7.0)$ \\
\hline \multicolumn{4}{|c|}{ Satisfied with current eating habits (C4) } \\
\hline Very satisfied & $101(20.2)$ & $53(20.5)$ & 48 (19.8) \\
\hline Satisfied & $198(39.5)$ & $112(43.2)$ & $86(35.5)$ \\
\hline Neither satisfied or dissatisfied & $162(32.3)$ & $77(29.7)$ & $85(35.1)$ \\
\hline Dissatisfied & $29(5.8)$ & $13(5.0)$ & $16(6.6)$ \\
\hline Very dissatisfied & $11(2.2)$ & $4(1.5)$ & $7(2.9)$ \\
\hline \multicolumn{4}{|c|}{ Top 5 factors/ persons who can persuade you to change eating habits (C5) ${ }^{\mathrm{a}}$} \\
\hline Family & $299(59.7)$ & $151(58.3)$ & $148(61.2)$ \\
\hline Doctor & 245 (48.9) & 115 (44.4) & $130(53.7)^{\star}$ \\
\hline Self & $187(37.3)$ & 107 (41.3) & $80(33.1)$ \\
\hline Friends & 165 (32.9) & $85(32.8)$ & $80(33.1)$ \\
\hline Dietician & $109(21.8)$ & 49 (18.9) & $60(24.8)$ \\
\hline \multicolumn{4}{|l|}{ Ways to keep track of health $(\mathrm{C} 6)^{\mathrm{a}}$} \\
\hline Weight & $308(61.5)$ & $157(60.6)$ & $151(62.4)$ \\
\hline Counting steps & $117(23.4)$ & $68(26.3)$ & $49(20.2)$ \\
\hline Monitoring calorie intake & 218 (43.5) & 108 (41.7) & $110(45.5)$ \\
\hline Fitness level & $186(37.1)$ & $111(42.9)$ & $75(31.0)^{\star}$ \\
\hline Health/ Fitness app & $146(29.1)$ & $85(32.8)$ & $61(25.2)$ \\
\hline Don't track health & $34(6.8)$ & $14(5.4)$ & $20(8.3)$ \\
\hline
\end{tabular}

\section{TABLE 5: Lifestyle Attributes of Survey Participants}

The relationship of demographic factors and lifestyle was evaluated by calculating odds ratios. The results showed that the participants with older age groups, 35 to 45 years, and 55 years and higher in comparison to the individuals in age groups 16 to 24 years, were more likely to have had a diabetes screening test with the 


\section{Cureus}

odds ratios of $2.2(\mathrm{CI}=1.3-3.7, \mathrm{P}=0.003)$ and $4.1(\mathrm{CI}=2.1-8.2, \mathrm{P}<0.001)$; respectively. Females were more likely to receive screening compared with males $(\mathrm{OR}=1.7, \mathrm{CI}=1.2-2.5, \mathrm{P}=0.003)$ (Table 6 ). Furthermore, married $(\mathrm{OR}=3.0, \mathrm{CI}=2.0-4.6, \mathrm{P}<0.001)$ and widowed/divorced participants ( $\mathrm{OR}=3.7, \mathrm{CI}=1.3-10.5, \mathrm{P}=0.01$ ) were more likely to obtain screening compared to singles. It was also found that the females were less likely to be physically active compared with men who were active $1-3$ times a week ( $\mathrm{OR}=0.64, \mathrm{CI}=0.41-0.99, \mathrm{P}=0.04)$. Qatari citizens were less physically active in both categories i.e. $1-3$ times/wk $(\mathrm{P}<0.001)$ and $4-7$ times/wk $(\mathrm{P}<0.001)$ compared with non-Qatari participants. Furthermore, Qatari nationals were also less likely to have healthy eating habits $(\mathrm{OR}=0.29, \mathrm{CI}=0.19-0.43, \mathrm{P}<0.001)$ compared with non-Qataris (Table 6$)$. The

participants who were physically active $4-7$ times per week $(\mathrm{OR}=0.20, \mathrm{CI}=0.08-0.49, \mathrm{P}<0.001)$ and eating healthy $(\mathrm{OR}=0.45, \mathrm{CI}=0.24-0.82, \mathrm{P}=0.009)$ were less likely to report that they were diagnosed with diabetes. 


\begin{tabular}{|c|c|c|c|c|c|c|c|c|c|}
\hline \multirow[t]{3}{*}{ Profile } & \multicolumn{3}{|c|}{ Screened for diabetes } & \multicolumn{4}{|c|}{ Frequency of exercise } & \multicolumn{2}{|l|}{ Healthy eating } \\
\hline & & & \multirow[b]{2}{*}{$P$ value } & \multicolumn{2}{|l|}{$1-3$ times a week } & \multicolumn{2}{|c|}{ 4-7 times a week } & \multirow[b]{2}{*}{ OR (95% Cl) } & \multirow[b]{2}{*}{ P value } \\
\hline & \multicolumn{2}{|c|}{ OR (95 \% Cl) } & & OR (95 \% Cl) & Pvalue & OR (95 \% Cl) & $P$ value & & \\
\hline \multicolumn{10}{|l|}{ Age groups } \\
\hline $16-24$ & Ref. & & & Ret. & & Ret. & & Ref. & \\
\hline $25-34$ & $\begin{array}{l}1.1 \\
1.9)\end{array}$ & (0.64, & 0.73 & $0.56(0.29,1.1)$ & 0.07 & $\begin{array}{l}0.79(0.40, \\
1.5)\end{array}$ & 0.48 & $\begin{array}{l}0.97(0.58, \\
1.6)\end{array}$ & 0.92 \\
\hline $35-54$ & $\begin{array}{l}2.2 \\
3.7)\end{array}$ & $(1.3$ & $0.003^{\star}$ & $0.64(0.34,1.2)$ & 0.17 & $\begin{array}{l}0.50(0.25, \\
1.0)\end{array}$ & $0.05^{\star}$ & $\begin{array}{l}0.93(0.56 \\
1.5)\end{array}$ & 0.79 \\
\hline $55+$ & $\begin{array}{l}4.1 \\
8.2)\end{array}$ & (2.1, & $<0.001^{*}$ & $0.85(0.37,1.9)$ & 0.7 & $\begin{array}{l}0.85(0.35 \\
2.1)\end{array}$ & 0.72 & $1.2(0.61,2.3)$ & 0.6 \\
\hline \multicolumn{10}{|l|}{ Gender } \\
\hline Male & Ref. & & & Ref. & & Ref. & & Ref. & \\
\hline Female & $\begin{array}{l}1.7 \\
2.5)\end{array}$ & $(1.2$ & $0.003^{*}$ & $0.64(0.41,0.99)$ & $0.04^{\star}$ & $\begin{array}{l}0.77(0.48, \\
1.2)\end{array}$ & 0.28 & $\begin{array}{l}0.84 \text { (0.58, } \\
1.2)\end{array}$ & 0.38 \\
\hline \multicolumn{10}{|l|}{ Nationality } \\
\hline Qatari & 1.5 & $(1.1,2.2)$ & $0.02^{\star}$ & $0.31(0.19,0.49)$ & $<0.001^{*}$ & $\begin{array}{l}0.34(0.21 \\
0.56)\end{array}$ & $<0.001^{*}$ & $\begin{array}{l}0.29 \text { (0.19, } \\
0.43)\end{array}$ & $<0.001^{*}$ \\
\hline Non-Qatari & Ref. & & & Ref. & & Ref. & & Ref. & \\
\hline \multicolumn{10}{|l|}{ Marital status } \\
\hline Married & 3.0 & $(2.0,4.6)$ & $<0.001^{\star}$ & $0.73(0.45,1.1)$ & 0.19 & $\begin{array}{l}0.58(0.35, \\
0.97)\end{array}$ & $0.04^{*}$ & $1.1(0.77,1.7)$ & 0.5 \\
\hline Single & Ref. & & & Ref. & & Ref. & & Ref. & \\
\hline $\begin{array}{l}\text { Widowed/ } \\
\text { divorced }\end{array}$ & $\begin{array}{l}3.7 \\
10.5\end{array}$ & (1.3, & $0.01^{*}$ & $0.64(0.18,2.1)$ & 0.46 & $\begin{array}{l}0.45(0.11, \\
1.8)\end{array}$ & 0.25 & $\begin{array}{l}0.68(0.24, \\
1.9)\end{array}$ & 0.46 \\
\hline \multicolumn{10}{|l|}{ Income group } \\
\hline 1,500-9,999 QAR & $\begin{array}{l}0.41 \\
.70)\end{array}$ & (0.23, & 0.001 & $7.4(3.2,17.3)$ & $<0.001^{\star}$ & $4.9(2.0,11.9)$ & $<0.001^{*}$ & $4.7(2.5,8.9)$ & $<0.001^{\star}$ \\
\hline $\begin{array}{l}10,000-19,999 \\
\text { QAR }\end{array}$ & 0.57 & $(0.35, .91)$ & 0.01 & $1.9(1.1,3.4)$ & $0.01^{*}$ & $1.1(0.64,2.2)$ & 0.58 & $1.1(0.74,1.8)$ & 0.47 \\
\hline 20,000+ QAR & Ref. & & & Ref. & & Ref. & & Ref. & \\
\hline \multicolumn{10}{|c|}{ Diagnosed with Diabetes(B1) } \\
\hline Yes & & & & $\begin{array}{l}0.79 \\
1.5)\end{array} \quad(0.40$ & 0.49 & $\begin{array}{l}0.20(0.08, \\
0.49)\end{array}$ & $<0.001^{*}$ & $\begin{array}{l}0.45(0.24, \\
0.82)\end{array}$ & $0.009^{\star}$ \\
\hline No & & & & Ret. & & Ret. & & Ref. & \\
\hline * significant at $5 \%$ & evel & & Reterenc & e tor Frequency of & Xercise: V & Ithout exercise & & & \\
\hline
\end{tabular}

TABLE 6: Relationship of Demographic Factors with Lifestyle and Diabetes Diagnosis

\section{Discussion}

The State of Qatar's National Health Strategy considers diabetes as one of the high-priority diseases for preventive healthcare [5]. Our study aimed at assessing the awareness and perception among the Qatar population including Qatari nationals and expatriates. Increased awareness among the population is a powerful step towards prevention of diabetes as it helps to assess causes, risks and complications and prompt people at risks to seek treatment and care. 
The study revealed that about $92 \%$ of respondents knew about at least one type of diabetes. Our results may not be comparable to other studies due to the unique methodology and location; however, in somewhat similar Indian study about $75 \%$ of the respondents had general awareness about diabetes [10]. In another study from an Ethiopian community showed that about $52.5 \%$ of the study participants were knowledgeable about diabetes [11]. Moreover, two other studies describe lack of knowledge pertaining to diabetes for example; a study conducted in Malaysia showed that around $58.1 \%$ of respondents had poor diabetes knowledge score among rural adult community [12]. Another Indian study, involving outpatients and inpatients showed that about $46 \%$ of diabetic patients had poor knowledge whereas interestingly $64 \%$ of non-diabetics had good knowledge regarding diabetes [13].

While examining the perceptions about risk factors leading to diabetes, majority of respondents considered hereditary factors (73.9\%), obesity (73.9\%) followed by pancreatic disease/injury (62.7\%), lack of exercise (61.7\%) and poor diet (61.3\%) as causative reasons in the development of the disease. These findings are somewhat consistent with the results of an Indian study where respondents considered family history of diabetes, consumption of more sweets, lack of exercise and obesity as main reasons of diabetes [10]. This is also congruent to the survey results done in a semi-urban Omani population, which identified obesity, physical inactivity and family history of diabetes as the top risk factors of diabetes perceived by the respondents [14]. In Qatar's case of T2DM for instance, a recent forecast of diabetes in Qatar highlighted obesity as the main driver of the rising diabetes prevalence in the country. About $60 \%$ of type 2 diabetes mellitus (T2DM) burden was ascribed to obesity while 10\% was attributed to smoking and physical inactivity combined [15].

While examining respondents' awareness about complications of diabetes, blindness was highly perceived as the most common outcome. Moreover, the majority of respondents agreed upon the fact that diabetes could cause amputation, kidney problems, and cardiovascular disease with a median response of 4 for these conditions. This finding is congruent to a study conducted in Oman whereby respondents identified the visual problem as the top complication, with heart disease, kidney disease, and wound infections as other complications [14]. In other studies, the respondents also identified foot problems, kidney disease, eye disease and cardiovascular diseases as complications of diabetes which is somewhat consistent with our findings $[14,16]$.

In our study, $12 \%$ of responded stated that they had diabetes with a higher proportion among females and Qatari nationals. This finding is comparable with the other existing data and studies from Qatar [4, 7, 9]. Moreover, the results from 2012 STEPwise survey in Qatar showed that about $12.7 \%$ of respondents were diagnosed with diabetes by a doctor or other health worker in past 12 months which is very close to our finding [17].

One of the challenges identified in our study was a relatively low screening proportion among participants and the lack of awareness about it. The results indicated that the lack of screening was mainly attributed to the fact that participants never thought about it. The main reasons for obtaining screening were a referral by health care provider and convenience of the process. Participants of older age groups, females, and people who were ever married were found more likely to be screened compared to their counterpart groups. This could be attributed to the fact that age is a risk factor for diabetes and females are mostly screened for gestational diabetes at the time of their first prenatal visit especially if they have existing risk factors. Gestational diabetes, which affects an estimated 18 percent of women during pregnancy, is caused by a change in the way a woman's body responds to insulin during pregnancy could be a major risk factor for the development of T2DM [18]. According to Kim et al, the only factors consistently associated with better screening rates were marital status and visit with an endocrinologist after delivery which increases the chances of screening among mothers especially who are perceived to be at higher risk [19]. Failure to screen for T2DM could result in missed opportunities for disease prevention [19]. Moreover, McCaig highlighted that marriage can provide a positive encouragement or support for a partner to be health conscious and to practice a healthy lifestyle [20].

In this study, the public hospital system was identified as the most preferred location for diabetes care in situations where respondents had any associated symptoms. This shows the important role and ease of accessibility of public sector health services in Qatar. The State of Qatar provides health services to all citizens and residents through government medical facilities through a health card system [21]. The system allows residents and citizens to obtain health services at various public healthcare facilities at no cost or at subsidized rates depending upon the level of care. Furthermore, to support the rising encounters in the health sector an increase in the number of healthcare facilities and workforce has been prioritized [22]. This effort ensures sufficient capacity in the system and health infrastructure in place in the future.

An important finding of this study was that the physical activity was relatively low with $25 \%$ of respondents reporting that they did not perform any exercise. The proportion of low physical activity was higher among female participants and consistent with the findings from previously completed studies in Qatar and regionally in Kuwait [4, 7, 23]. It was also evident that the Qatari nationals and females (Qatari and nonQatari) were less likely to be physically active compared to non-Qataris and males (Qatari and non-Qatari), respectively. About $57 \%$ of respondents considered their eating habits healthy, $60 \%$ were satisfied or very satisfied with their eating habits. The family persuasion was the major factor in changing eating habits as 
evident by another study, which supports the fact the family and social environments could play an important role in the determination of dietary patterns and quality among children [24]. In our study, the people who were physically active and eating healthy were less likely to have had diabetes. This provides evidence that lifestyle modifications such as physical activity can delay the onset or prevent diabetes. To improve public health and further understand the underlying mechanisms behind findings of this study, the surveillance and mixed method approaches (quantitative and qualitative) can be used to study chronic diseases including diabetes and related risk factors especially the ones already exist in Qatar [4, 8-9, 25-28].

\section{Conclusions}

One implication of this study is that the general population in Qatar require more awareness and education regarding diabetes prevention and risk factors. Behaviors towards diabetes diagnosis and prevention vary by demographic attributes. It is more likely for older age group, females, Qatari nationals, and married/widowed/divorced to get screened for diabetes; more likely for males, non-Qataris, younger and single people to indulge in exercise; and more likely for non-Qataris to practice healthy eating habits than their counterparts. This demonstrates a need to implement more campaigns to encourage healthier lifestyles. The findings of this study will specifically useful for the Qatar's National Diabetes and Health strategies in further strengthening ongoing and future programs through evidence based approaches.

\section{Additional Information}

\section{Disclosures}

Human subjects: Consent was obtained by all participants in this study. Approved by MoPH issued approval N/A. All ethical procedures were followed during data collection process as per accordance with the Ministry of Public Health (MoPH) policies. This work was supported and approved by MoPH. . Animal subjects: All authors have confirmed that this study did not involve animal subjects or tissue. Conflicts of interest: In compliance with the ICMJE uniform disclosure form, all authors declare the following: Payment/services info: All authors have declared that no financial support was received from any organization for the submitted work. Financial relationships: All authors have declared that they have no financial relationships at present or within the previous three years with any organizations that might have an interest in the submitted work. Other relationships: All authors have declared that there are no other relationships or activities that could appear to have influenced the submitted work.

\section{Acknowledgements}

The authors declare no conflict of interest. All ethical procedures were followed during data collection process as per accordance with the MOPH policies. The participants have consented before the survey and parental consent was sought for respondents aged below 18-year-old. This work was supported and funded by MOPH. We thank Hamad Medical Corporation, Qatar, Primary Health Care Corporation and YouGov for their support in this project.

\section{References}

1. World Health Organization. Diabetes. (2017). Accessed: March 9, 2017: http://www.who.int/mediacentre/factsheets/fs312/en/.

2. World Health Organization. EMRO. Diabetes. Health topics . (2017). Accessed: March 9, 2017: http://www.emro.who.int/health-topics/diabetes/index.html.

3. Badran M, Laher I: Obesity in Arabic-speaking countries. J Obes. 2011, 2011:1-9.

4. Al-Thani MH, Al-Thani AA, Al-Chetachi WF, et al.: Dietary and nutritional factors influencing obesity in Qatari adults and the modifying effect of physical activity. J Obes Weight-Loss Medic. 2015, 1:1-7.

5. State of Qatar, Ministry of Public Health. National Health Strategy 2011-2016 . (2018). Accessed: January 8, 2018: https://www.moph.gov.qa/HSF/Documents/short\%20report\%20eng\%2020.03.2018.pdf.

6. Middle East forum on quality and safety in healthcare, National Diabetes Strategy updates . (2018). Accessed: May 16, 2018: https://www.hamad.qa/EN/AllEvents/MEFQSH2018/Documents/Sunday/BreakoutGroupC/C10-2_MEF2018\%20-Dr.\%20AlAnoud_PPT_Fin....

7. Al-Thani MH, Cheema S, Sheikh J, et al.: Prevalence and determinants of metabolic syndrome in Qatar: results from a National Health Survey. BMJ Open. 2016, 6:e009514. Accessed: May 13, 2018: 10.1136/bmjopen-2015-009514

8. Ali FM, Nikoloski Z, Reka H, Gjebrea O, Mossialos E: The diabetes-obesity-hypertension nexus in Qatar: evidence from the World Health Survey. Popul Health Metr. 2014, 12:18. Accessed: May 13, 2018: 10.1186/1478-7954-12-18

9. Al-Thani M, Al-Thani AA, Al-Chetachi W, et al.: Situation of diabetes and related factors among Qatari adults: findings from a community-based survey. JMIR Diabetes. 2017, 2:e7. 10.2196/diabetes.7535

10. Mohan D, Raj D, Shanthirani CS, et al.: Awareness and knowledge of diabetes in Chennai--the Chennai Urban Rural Epidemiology Study [CURES-9]. J Assoc Physicians India. 2005, 53:283- 7.

11. Kassahun CW, Mekonen AG: Knowledge, attitude, practices and their associated factors towards diabetes mellitus among non-diabetes community members of Bale Zone administrative towns, South East Ethiopia. A cross-sectional study. PLoS ONE. 2017, 12:e0170040. Accessed: May 13, 2017:

10.1371/journal.pone.0170040

12. Minhat HS, Hamedon TR: Understanding towards diabetes mellitus among rural adult community in 
Malaysia. World J Med Sci. 2014, 11:217-221.

13. Konduru SS, Ranjan A, Karthik SM, Shaik S, Vakkapatla LS: Assessment of diabetes related knowledge, attitude and practice among diabetics and non-diabetics using self prepared questionnaire for awareness of health promotion. Indian J of Pharm Pract. 2017, 10:32-38. 10.5530/ijopp.10.1.8

14. Al Shafaee MA, Al-Shukaili S, et al.: Knowledge and perceptions of diabetes in a semi-urban Omani population. BMC Public Health. 2008, 8:249. Accessed: May 13, 2018: 10.1186/1471-2458-8-249

15. Awad SF, O'Flaherty M, Critchley J, Abu-Raddad LJ: Forecasting the burden of type 2 diabetes mellitus in Qatar to 2050: A novel modeling approach. Diabetes Res Clin Pract. 2018, 137:100-108. 10.1016/j.diabres.2017.11.015

16. Muninarayana C, Balachandra G, Hiremath SG, Iyengar K, Anil NS: Prevalence and awareness regarding diabetes mellitus in rural Tamaka, Kolar. Int J Diabetes Dev Ctries. 2010, 30:18-21.

17. Ministry of Public Health. STEPwise report for chronic disease risk factor surveillance . (2012). Accessed: May 6, 2017: https://www.mdps.gov.qa/en/statistics/Surveys/STEPwise_Report.pdf.

18. American Diabetes Association. American Diabetes Association Issues Updated Diabetes and Hypertension Position Statement. (2017). Accessed: January 6, 2018: http://www.diabetes.org/newsroom/pressreleases/2017/american-diabetes-association-updated-diabetes-and-hypertension-....

19. Kim C, Tabaei BP, Burke R, et al.: Missed opportunities for type 2 diabetes mellitus screening among women with a history of gestational diabetes mellitus. Am J Public Health. 2006, 96:1643-1648. 10.2105/AJPH.2005.065722

20. Divorced people more likely to die from preventable accidents than married people. Rice University News \& Media. (2013). Accessed: January 8, 2018: http://news.rice.edu/2013/10/30/divorced-people-more-likely-todie-from-preventable-accidents-than-married-people-2/.

21. Hamad Medical Corporation. How to get a health card?. (2017). Accessed: July 16, 2017: https://www.hamad.qa/EN/Patient-Information/How-To-Get-A-Health-Card/Pages/default.aspx.

22. Qatar Healthcare Facilities Master Plan 2013-2033. (2014). Accessed: April 8, 2018: https://www.moph.gov.qa/ar/health-strategies/Documents/QHFMP\%20-\%20Report.pdf.

23. EMAN. Eastern Mediterranean approach for control of non communicable diseases. Survey of risk factors for chronic non communicable diseases. State of Kuwait. (2015). Accessed: July 16, 2017: http://www.who.int/chp/steps/Kuwait_2014_STEPS_Report.pdf?ua=1.

24. Patrick H, Nicklas TA: A review of family and social determinants of children's eating patterns and diet quality. J Am Coll Nutr. 2005, 24:83-92.

25. Stone K, Horney JA: Methods: surveillance. Disaster Epidemiology: Methods and Applications. Academic Press, London; 2018. 11-23. 10.1016/B978-0-12-809318-4.00002-2

26. Al-Thani M, Al-Thani A, Al-Chetachi W, Akram H: Obesity and related factors among children and adolescents in Qatar. Int J Basic Sci Med. 2017, 2:161-165.

27. Al-Thani M, Al-Thani A, Alyafei S, et al.: The prevalence and characteristics of overweight and obesity among students in Qatar [epub ahead of print]. Public Health. 2018, 10.1016/j.puhe.2018.03.020

28. Al-Thani M, Al-Thani A, Alyafei S, et al.: Prevalence of physical activity and sedentary-related behaviors among adolescents: data from the Qatar National School Survey [epub ahead of print]. Public Health. 2018, 10.1016/j.puhe.2018.03.019 